\title{
SOME PRECISIONS ON THE FOURIER-BOREL TRANSFORM AND INFINITE ORDER DIFFERENTIAL EQUATIONS

\author{
by LAWRENCE GRUMAN
}

(Received 21 February 1972; revised 31 August, 1972)

Let $f(z)$ be an entire function (of several variables). We define the function

$$
M_{f}(r)=\max _{\|z\|=r}|f(z)|,
$$

which is increasing. The order of $f(z)$ is the constant (perhaps infinite)

$$
\rho=\varlimsup_{r \rightarrow \infty} \frac{\log \log M_{f}(r)}{\log r} .
$$

If $\rho<+\infty$, we define a proximate order as a function $\rho(r)$ such that

(1) $\rho(r) \rightarrow \rho$ and $\rho^{\prime}(r) r \log r \rightarrow 0$ as $r \rightarrow \infty$.

We can also assume the additional condition

(2) $\rho^{\prime \prime}(r) r^{2} \log r \rightarrow 0 \quad$ as $r \rightarrow \infty$.

If $L(r)=r^{\rho(r)-\rho}$, then we have

(3) $\lim _{r \rightarrow \infty} \frac{L(k r)}{L(r)}=1$ uniformly for $0<a \leqq k \leqq b<+\infty$.

We define the type of $f(z)$ with respect to $\rho(r)$ by

$$
\sigma=\varlimsup_{r \rightarrow \infty} \frac{\log M_{f}(r)}{r^{\rho(r)}}
$$

and $f(z)$ is said to be of (a) minimal, (b) normal, or (c) maximal type if (a) $\sigma=0$, (b) $0<\sigma<+\infty$, or (c) $\sigma=+\infty$, respectively. For every function $f(z)$ of order $\rho$, there exists a proximate order $\rho(r)$ such that $f(z)$ has normal type with respect to $\rho(r)[4]$.

If $\rho>1$, we assume that $\rho(r)>1$ and $(d / d r)\left(r^{\rho(r)-1}\right)>0$ for all $r$. Since this holds eventually, this assumption involves no loss of generality. Then the equation $t=r^{\rho(r)-1}$ has a unique solution $r$ for all $t \geqq 0$. We define the dual proximate order $\rho^{*}(t)$ by $\rho^{*}(t)=$ $\rho(r) /(\rho(r)-1)$, where $r$ is this unique solution. It is an easy calculation to check that $\rho^{*}(t)$ satisfies (1) and (2) and that $\rho^{* *}(r)=\rho(r)$.

For a real-valued continuous function $q(z)$, we define the Banach space $B_{q}$ to be the set of entire functions such that

$$
|f(z) \exp (-q(z))| \rightarrow 0 \text { as }\|z\| \rightarrow \infty,
$$


where the norm in $B_{q}$ is the sup norm. If $q_{n}(z)$ is a decreasing (resp. increasing) sequence of functions, we define the set $F=\bigcap B_{q_{n}}$ (resp. $E=\bigcup B_{q_{n}}$ ), which we equip with the projective limit (resp. inductive limit) topology. We designate the dual space of continuous linear functionals by $F^{\prime}$ (resp. $E^{\prime}$ ). $F$ is a Fréchet space, and $E^{\prime}$ can be given the topology of a Fréchet space under the norm topologies

$$
\|v\|_{n}=\sup _{\substack{f \in B_{q_{n}} \\\|f\| \|_{n}=1}}|v(f)|
$$

In particular, let $p(z)$ be a pseudonorm (i.e., $p\left(z_{1}+z_{2}\right) \leqq p\left(z_{1}\right)+p\left(z_{2}\right), p(t z)=t p(z)$ for $t \geqq 0)$ and let $p_{n}(z)=p(z)+\|z\| / n$ with $p_{n}^{\prime}(u)=\sup _{p_{n}(z) \leqq 1} \operatorname{Re}\langle u, z\rangle$ be the dual norm. We designate by $F_{p}^{\rho(r)}$ (resp. $\left.E_{p^{\prime}}^{\rho(r)}\right)$ the space we get by taking $q_{n}(z)=\left(p_{n}(z)\right)^{\rho(\| z||)}$ (resp. $\left.q_{n}(z)=\left(p_{n}^{\prime}(z)\right)^{\rho(\| z|z| \mid)}\right)$. It is clear from (3) that we can replace $q_{n}(z)$ by $q_{n}^{\prime}(z)=\left(p_{n}(z)\right)^{\rho\left(p_{n}(z)\right)}$ (resp. $\left.\left(p_{n}^{\prime}(z)\right)^{\rho\left(p_{n}^{\prime}(z)\right)}\right)$ and still obtain the same topological vector spaces. For $v \in\left(F_{p}^{\rho(r)}\right)^{\prime}$ (resp. $\left.\left(E_{p^{\prime}}^{\rho(r)}\right)^{\prime}\right)$, we define $f_{v}(u)$ by

$$
f_{v}(u)=v(\exp \langle u, z\rangle)
$$

which is an entire function of $u$, called the Fourier-Borel transform of $v$.

In [7], A. Martineau showed that, if $\rho>1$ is a constant and $p(z)$ is a complex norm (i.e., $p(\lambda z)=|\lambda| p(z)$ ), then the Fourier-Borel transform establishes an isomorphism between the spaces $\left(F_{p}^{\rho}\right)^{\prime}$ and $E_{\tau p^{\prime}}^{\rho *}$ (for a suitable constant $\tau$ ). He introduced the notion of a constant coefficient differential operator of infinite order $\check{\alpha}$, and, using the Fourier-Borel transform, showed that, for every $f \in F_{p}^{\rho}$, the equation $\check{\alpha}(x)=f$ has a solution $g \in F_{p}^{\rho}$. In [1], the author extended this result to the case of complex pseudonorms and proximate orders (as well as to the case $\rho<1$ ).

We shall be primarily interested here in showing that the isomorphism proven by Martineau is still valid for arbitrary pseudonorms (not necessarily complex), for $\rho>1$, and for all proximate orders. It is then a simple matter to apply his reasoning to the case of differential equations of infinite order in order to get a more precise estimate of the growth of solutions.

Before turning to the main theorems, we first collect some results which we shall need later.

Proposition 1. Let $E, F$ be two Fréchet spaces and $\beta$ a continuous linear map of $E$ into $F$. The two following statements are equivalent:

(i) $\beta$ is onto.

(ii) ${ }^{t} \beta: F^{\prime} \rightarrow E^{\prime}$ (the transpose map) is one-to-one and its image ${ }^{\imath} \beta\left(F^{\prime}\right)$ is weakly closed in $E^{\prime}$.

Proof. See [8].

PROPOSTIION 2. Every element of the dual space of $F_{p}^{\rho(r)}$ can be represented by integration with respect to a measure $\mu$ such that 
(5) $\quad \dot{\mu} \cdot \exp \left(p_{n}(z)\right)^{\rho(\|z\|)}$ is a bounded measure for some $n$.

Every element of the dual space of $E_{p^{\prime}}^{\rho(r)}$ can be represented by integration with respect to a measure $\mu$ such that

(6) $\mu \cdot \exp \left(p_{n}^{\prime}(z)\right)^{\rho(\|=1\|)}$ is a bounded measure for all $n$.

(The representations are not unique.)

Proof. The proof can be found in [7].

COROLlARY. If we equip $\left(E_{p^{\prime}}^{\rho(r)}\right)^{\prime}$ with its Fréchet space topology, then $\left(\left(E_{p^{\prime}}^{\rho(r)}\right)^{\prime}\right)^{\prime}=E_{p^{\prime}}^{\rho(r)}$.

Proof. The dual space is clearly a family of functions containing $E_{p^{\prime}}^{\rho(r)}$, and, by considering the Dirac measures associated with every point, it is clear that every function $h(z)$ in the dual satisfies the condition

$$
\sup _{z}\left|h(z) \exp \left(-q_{n}(z)\right)\right| \leqq M<+\infty
$$

for $n$ sufficiently large. Thus it remains to show that $h(z)$ is holomorphic.

For a given complex line $u \lambda$ through the point $z$, we let $\gamma$ be a rectifiable closed compact curve in $u \lambda$ and $\alpha$ represent integration around $\gamma$. Then $\alpha(f)=0$ for every $f \in E_{p^{\prime}}^{\rho(r)}$; so $\alpha(h)=0$ for every $h \in\left(\left(E_{p^{\prime}}^{\rho(r)}\right)^{\prime}\right)^{\prime}$. Thus $h$ is holomorphic in every complex line through $z$ and hence holomorphic in $\mathbb{C}^{n}$.

LEMMA. Let $\rho(r)$ be a proximate order, with $\rho>1$. If $\eta(r)$ is a nonnegative function such that $\lim _{r \rightarrow \infty} \eta(r) r^{-\rho(r)}=0$, there exists a positive function $\xi(r)$ with nonnegative first and second derivatives such that $\xi(r) \geqq \eta(r)$ and $\lim _{r \rightarrow \infty} \xi(r) r^{-\rho(r)}=0$.

Proof. Let $\left\{\varepsilon_{n}\right\}$ be a decreasing sequence of positive numbers approaching zero and $\left\{r_{n}\right\}$ a sequence of numbers such that $\eta(r) \leqq \varepsilon_{n+1} r^{\rho(r)}$ for $r \geqq r_{n}$. We assume, without loss of generality, that both $d r^{\rho(r)} / d r$ and $d^{2} r^{\rho(r)} / d r^{2}$ are everywhere positive (by (1) and (2), this holds eventually).

We construct a function $\xi_{1}(r)$ to be piecewise linear. The construction will be carried out by induction. For $n=1$, we choose for $\xi_{1}(r)$ a constant such that $\xi_{1}(r)=\max \left(\eta(r), \varepsilon_{1} r^{\rho(r)}\right)$. Having constructed $\xi_{1}(r)$ for $r \leqq r_{n}$ with the property that $\xi_{1}(r) \geqq \varepsilon_{n-1} r^{\rho(r)}$ for $r_{n-1} \leqq r \leqq r_{n}$, we construct $\xi_{1}(r)$ for $r_{n} \leqq r \leqq r_{n+1}$. We continue $\xi_{1}(r)$ linearly unless there exists an $R_{n}$, with $r_{n} \leqq R_{n} \leqq r_{n+1}$, such that $\xi_{1}\left(R_{n}\right)=\varepsilon_{n-1} R_{n}^{\rho\left(R_{n}\right)}$. If this occurs, we continue $\xi_{1}(r)$ past $R_{n}$ by taking $\delta>0$ and taking the tangent to the curve $\varepsilon_{n-1} r^{\rho(r)}$ at $R_{n}$; at $R_{n}+q \delta$, for $q$ an integer, we extend this continuation as a continuous function by making a linear extension with slope $\left.(d / d r)\left\{\varepsilon_{n-1} r^{\rho(r)}\right\}\right|_{R_{n}+q \delta}$. By choosing $\delta$ sufficiently small, we shall have $\xi_{1}(r) \geqq \varepsilon_{n} r^{\rho(r)}$ in the interval $r_{n} \leqq r \leqq r_{n+1}$. This establishes the induction. Furthermore, it is clear that $\xi_{1}(r) \geqq \eta(r)$, and that, given $n$, for $r$ sufficiently large, $\xi_{1}(r) \leqq \varepsilon_{n} r^{(r)}$.

Let $\alpha(r)$ be a nonnegative $C^{\infty}$ function with compact support depending only on $|r|$, such that $\int \alpha(r) d r=1$. Then $\xi(r)=\int \xi_{1}\left(r^{\prime}\right) \alpha\left(r-r^{\prime}\right) d r^{\prime}$ satisfies the requirements of the lemma. 
THEOREM 1. The Fourier-Borel transform given by (4) establishes an isomorphism between the spaces

(i) $\left(F_{p}^{\rho(r)}\right)^{\prime}$ and $E_{\tau p}^{\rho *(r)}$,

and between the spaces

(ii) $\left(E_{p^{\prime}}^{\rho(r)}\right)^{\prime}$ and $F_{\tau p}^{\rho^{*}(r)}$,

where

$$
\tau=\frac{\rho}{(\rho-1)^{(\rho-1) / \rho}}
$$

Proof. Let $v \in\left(F_{p}^{\rho(r)}\right)^{\prime}$. Then, by Proposition 2, there exists an $n$ such that

$$
|v(f)| \leqq C_{v} \sup _{z}\left|f(z) \exp \left(-p_{n}(z)\right)^{\rho\left(p_{n}(z)\right)}\right|,
$$

for some constant $C_{v}$. Thus

$$
\begin{aligned}
\left|f_{v}(u)\right| & \leqq C_{v} \sup \left|\exp \langle u, z\rangle-\left(p_{n}(z)\right)^{\rho\left(p_{n}(z)\right)}\right| \\
& \leqq C_{v} \exp \left(\sup _{t \geq 0}\left(\sup _{p_{n}(z)=1}\{\operatorname{Re}\langle u, z\rangle\} t-t^{\rho(t)}\right)\right) \\
& \leqq C_{v} \exp \left(\sup _{t \geqq 0} p_{n}^{\prime}(u) t-t^{\rho(t)}\right) .
\end{aligned}
$$

Now

$$
\frac{d}{d t}\left(p_{n}^{\prime}(u) t-t^{\rho(t)}\right)=p_{n}^{\prime}(u)-\left(\rho^{\prime}(t) \log t+\frac{\rho(t)}{t}\right) t^{\rho(t)} .
$$

It follows from (1) that, for large values of $\|u\|$, this function takes on an absolute maximum.

For arbitrary $\delta>0$, it follows from (1) that, for $\|u\|$ sufficiently large (depending on $\delta$ ), the maximum occurs at $t_{u}^{\rho\left(t_{u}\right)-1}=p_{n}^{\prime}(u) /(\rho+\xi(u))$ for $|\xi(u)|<\delta$ and equals

$$
p_{n}^{\prime}(u)^{\rho\left(t_{u}\right) /\left(\rho\left(t_{u}\right)-1\right)}\left\{\left(\frac{1}{\rho+\zeta(u)}\right)^{1 /\left(\rho\left(t_{u}\right)-1\right)}-\left(\frac{1}{\rho+\zeta(u)}\right)^{\rho\left(t_{u}\right) /\left(\rho\left(t_{u}\right)-1\right)}\right\},
$$

which is less than or equal to

$$
p_{n}^{\prime}(u)^{\rho^{*}\left(k(u) p_{n}{ }^{\prime}(u)\right)}(\tau+\varepsilon),
$$

where $\varepsilon \rightarrow 0$ as $\delta \rightarrow 0$ and $0<a \leqq k(u) \leqq b<\infty$. So the maximum for large $\|u\|$ is less than $\tau p_{n+1}^{\prime}(u)^{\rho^{*}\left(p_{n}{ }^{\prime}+1(u)\right)}$ by (3). Thus the mapping $v \rightarrow f_{v}$ of $\left(F_{p}^{\rho(r)}\right)^{\prime}$ is into $E_{\tau p^{\prime}}^{\rho^{*}(r)}$. Similarly, one shows that the mapping $v \rightarrow f_{v}$ of $\left(E_{p^{\prime}}^{\rho(r)}\right)^{\prime}$ is into $F_{\tau p}^{\rho^{*}(r)}$.

To show that the mappings are onto, we use an adaptation of an argument of Hörmander [2, p. 100]. Let $K=\left\{z: \operatorname{Re}\langle u, z\rangle \leqq p(u), u \in \mathbb{C}^{N}\right\}$. We let $x$, a $2 N$-tuple, represent the real coordinates of $z$. We define

$$
\phi(v)=\sup _{x \in K}\left(x_{1} \operatorname{Im} v_{1}+\ldots+x_{2 N} \operatorname{Im} v_{2 N}\right)
$$


which is plurisubharmonic in the variable $v$. Then $\theta(v)=(\phi(v))^{\rho(\phi(v))}$ is also plurisubharmonic for $\phi(v)$ sufficiently large, for, given any complex line (which we assume, without loss of generality, to be the line $\left.\lambda\left(v_{1}, 0, \ldots, 0\right)\right)$, we have

$$
\begin{aligned}
\frac{d^{2} \theta(v)}{d v_{1} d \bar{v}_{1}}=\left(\rho^{\prime \prime}(\phi) \log \phi+2 \frac{\rho^{\prime}(\phi)}{\phi}\right. & -\frac{\rho(\phi)}{\phi^{2}} \\
+ & {\left.\left[\rho^{\prime}(\phi) \log \phi+\frac{\rho(\phi)}{\phi}\right]^{2}\right) \phi^{\rho(\phi)} \frac{d \phi}{d v_{1}} \frac{d \phi}{d \bar{v}_{1}} } \\
+ & \left(\rho^{\prime}(\phi) \log \phi+\frac{\rho(\phi)}{\phi}\right) \phi^{\rho(\phi)} \frac{d^{2} \phi}{d v_{1} d \bar{v}_{1}}
\end{aligned}
$$

By adjusting $\rho(r)$ on a bounded set of $r$, if necessary, we may assume that $\theta(v)$ is everywhere plurisubharmonic.

Let $F(u) \in F_{p}^{\rho(r)}$, and let $\eta(r)=\sup _{\|u\|=r}\left(\sup \left(\log |F(u)|-p(u)^{\rho(p(u))}, 0\right)\right)$. For $\varepsilon>0$, we let $p_{\varepsilon}(u)=\sup (p(u), \varepsilon\|u\|)$. Then, since $p_{\varepsilon}(u)$ is continuous and

$$
\varlimsup_{r \rightarrow \infty} \frac{\log |F(r u)|}{r^{\rho(r)}} \leqq p_{\varepsilon}(u)^{\rho},
$$

it follows from Hartog's Theorem applied to plurisubharmonic functions (cf. [5, 6, 3, Corollary to Theorem 5.4.1]) that, for the compact set $\|u\|=1$,

$$
\frac{\log |F(r u)|}{r^{\rho(r)}} \leqq p_{\varepsilon}(u)^{\rho}+\varepsilon
$$

for $r$ sufficiently large. This implies, by (3), that $\log |F(z)| \leqq p_{\varepsilon}(z)^{\rho\left(p_{c}(z)\right)}+\varepsilon\|z\|^{\rho(\|z\| \|)}$ for $\|z\|$ sufficiently large, which in turn implies that $\lim _{r \rightarrow \infty} \eta(r) r^{-\rho(r)}=0$. Thus, by the lemma, there exists a positive function $\xi(r)$ with nonnegative first and second derivatives such that $\lim _{r \rightarrow \infty} \xi(r) r^{-\rho(r)}=0$ and $\xi(r) \geqq \eta(r)$. Let $\phi^{*}(v)=\sup _{\|x\| \leqq 1}\left(x_{1} \operatorname{Im} v_{1}+\ldots+x_{2 N} \operatorname{Im} v_{2 N}\right)$ and $\xi\left(\phi^{*}(v)\right)=\xi^{*}(v)$, which is plurisubharmonic.

Let $\Sigma$ be the $N$-dimensional subspace, $v=\left(i u_{1},-u_{1}, \ldots, i u_{N},-u_{N}\right)$ and $w$ be the function $\left(i u_{1},-u_{1}, \ldots, i u_{N},-u_{N}\right) \rightarrow F\left(u_{1}, \ldots, u_{N}\right)$. Then $|w(v)| \leqq C_{0} \exp \left(\theta(v)+\xi^{*}(v)\right)$ on $\Sigma$. Thus, if $\theta^{\prime}(v)=\theta(v)+\xi^{*}(v)+\log \left(1+\|v\|^{2}\right)^{N}$, then $\int_{\Sigma}|w(v)|^{2} \exp \left(-2 \theta^{\prime}(v)\right) d \sigma(v)<\infty$, where $d \sigma$ indicates the Lebesgue measure.

By a modification of the proof of Theorem 4.4.3 of [2] (due to A. Martineau; cf. [5] or [3, Theorem 5.3.3]), we have the following result: If $\psi$ is a plurisubharmonic function in $\mathbb{C}^{m}$ and $f$ is holomorphic in $\mathbb{C}^{k}(k<m)$ such that $\int_{\mathbb{C}^{k}}|f|^{2} \exp (-\psi) d \sigma<\infty$, then there exists $g$, holomorphic in $\mathbb{C}^{m}$, such that $g=f$ on $\mathbb{C}^{k}$ and $\int_{\mathbb{C}^{m}}|g|^{2} \exp \left(-\psi^{\prime}\right)\left(1+\|z\|^{2}\right)^{-3(m-k)} d \sigma<\infty$, where $\psi^{\prime}(z)=\sup _{\left\|z^{\prime}-z\right\| \leqq 2(m-k)} \psi\left(z^{\prime}\right)$. 
Applying this to the present case, we can find an entire function $W$ in $\mathbb{C}^{2 N}$ such that $W=w$ on $\Sigma$ and

$$
\int|W(v)|^{2} \exp \left(-2 \theta^{\prime \prime}(v)\right)(1+\|v\|)^{-6 N} d \sigma(v)<\infty,
$$

where $\theta^{\prime \prime}(v)=\sup _{\left\|v-v^{\prime}\right\| \leqq 2 N} \theta^{\prime}(v)$. From this we conclude, by Schwarz's Lemma (cf. [5]), that there exists a constant $C_{0}^{\prime}$ such that

$$
|W(v)| \leqq C_{0}^{\prime}(1+\|v\|)^{3 N} \exp \theta^{\prime \prime \prime}(v),
$$

where $\theta^{\prime \prime \prime}(v)=\sup _{\left\|v-v^{\prime}\right\|<2 N+1} \theta^{\prime}\left(v^{\prime}\right)$, and hence, following the same reasoning as in [2, Theorem 4.5.3], there exists a function $W^{\prime}(v)$ such that $W^{\prime}(v)=w(v)$ on $\Sigma$ and

$$
\left|W^{\prime}(v)\right| \leqq C_{0}^{\prime \prime}(1+\|v\|)^{-2 N-1} \exp \left(\theta^{\prime \prime \prime}(v)+\varepsilon \sum_{i=1}^{2 N}\left|\operatorname{Im} v_{i}\right|\right) \text {. }
$$

By the Paley-Wiener Theorem, if

$$
\mu(x)=\frac{1}{(2 \pi)^{2 N}} \int_{R^{2 N}} \exp i\left\langle x, v+i v^{\prime}\right\rangle W^{\prime}\left(v+i v^{\prime}\right) d v,
$$

then $\mu(x)$ is continuous and independent of $v^{\prime}$, and the Fourier-Laplace transform of $\mu(x)$, $\int \exp \left\{-i\left(x_{1} v_{1}+\ldots+x_{2 N} v_{2 N}\right)\right\} \mu(x) d x=W^{\prime}(v)$; hence the Fourier-Borel transform of $\mu(x)$ is $F(u)$. In this case, it follows from (7) that

$$
\mu(x) \leqq K_{n} \exp \left(\inf _{u} p_{n}(u)^{\rho\left(p_{n}(u)\right)}-\operatorname{Re}\langle u, z\rangle\right)
$$

and, by applying the same reasoning as above, we conclude that

$$
\mu(x) \leqq K_{n}^{\prime} \exp \left\{-\tau p_{n-1}^{\prime}(u)^{\rho^{*}\left(p_{n-1}(u)\right)}\right\}
$$

for all $n$, which implies that $\mu(x)$ satisfies (6). Thus the map $\left(E_{\tau p^{\prime}}^{\rho^{*}(r)}\right)^{\prime} \rightarrow F_{p}^{\rho(r)}$ is onto and, since

$$
\tau^{*}=\frac{\rho^{*}}{\left(\rho^{*}-1\right)^{\left(\rho^{*}-1\right) / \rho^{*}}}=\frac{1}{\tau}
$$

(where $1 / \rho^{*}+1 / \rho=1$ ) and $\rho^{* *}(r)=\rho(r),\left(E_{p^{\prime}}^{\rho(r)}\right)^{\prime} \rightarrow F_{\tau p}^{\rho^{*}(r)}$ is onto. Similarly, one shows that the mapping of $\left(F_{p}^{\rho(r)}\right)^{\prime}$ into $E_{\tau p^{\prime}}^{\rho^{*}(r)}$ given by (4) is onto.

The map $v \rightarrow f_{v}$ is thus a continuous mapping of the Fréchet space $\left(E_{p^{\prime}}^{\rho(r)}\right)^{\prime}$ onto $F_{\tau p}^{\rho^{*}(r)}$, which implies, by Proposition 1, that the transpose map of $\left(F_{\tau p}^{\rho^{*}(r)}\right)^{\prime}$ into $E_{p^{\prime}}^{\rho(r)}$ is one-to-one with closed image. In fact, we know that the map of $\left(F_{\tau p}^{\rho^{*}(r)}\right)^{\prime}$ is onto $E_{p^{\prime}}^{\rho(r)}$, which implies in turn that the map of $\left(E_{p^{\prime}}^{\rho(r)}\right)^{\prime}$ is one-to-one onto $F_{\tau p}^{\rho^{*}(r)}$, which establishes the desired isomorphisms.

COROLLARY. In the space $F_{p}^{\rho(r)}$, the subspaces spanned by

(i) $\exp \langle u, z\rangle$ for $u \in K$ with $\stackrel{\circ}{K} \neq \emptyset$,

(ii) $z^{\alpha} \exp \left\langle u_{0}, z\right\rangle$ for all multi-indices of nonnegative integers $\alpha$, are dense (in particular, the exponentials and the polynomials are dense). 
Proof. For $v \in\left(F_{p}^{\rho(r)}\right)^{\prime}$, if $v(\exp \langle u, z\rangle)=0$ for $u \in K$, then $f_{v} \equiv 0$, from which (i) follows. The function $v\left(z^{\alpha} \exp \left\langle u_{0}, z\right\rangle\right)=c_{\alpha}$, where $c_{\alpha}$ is the coefficient of $\left(u-u_{0}\right)^{\alpha}$ in the Taylor's series expansion of $f_{v}$ at $u_{0}$. Thus, if $c_{\alpha}=0$ for all $\alpha$, then $f_{v} \equiv 0$, from which (ii) follows.

For $v \in F_{p}^{\rho(r)}$ such that $f_{v}$ has type zero (with respect to $\rho^{*}(r)$ ), we define the convolution

$$
v * \mu(f)=v_{w}\left[\mu_{v}(f(w+v))\right] .
$$

Then, by Theorem 1, the map $\mu \rightarrow v * \mu$ is a map of $\left(F_{p}^{\rho(r)}\right)^{\prime}$ into itself. We define a differential equation of infinite order to be

$$
(\check{v}(f), \mu)=(f, v * \mu) .
$$

THEOREM 2. If $v \in\left(F_{p}^{\rho(r)}\right)^{\prime}$ is such that $f_{v}$ has minimal type with respect to $\rho *(r)$, then the equation $\check{v}(x)=f$, for $f \in F_{p}^{\rho(r)}$, always has a solution in $F_{p}^{\rho(r)}$.

Proof. The reader is referred to [7]; the proof that Martineau gives there for complex norms easily carries over to the present case.

\section{REFERENCES}

1. L. Gruman, The growth of entire solutions of differential equations of finite and infinite order, Ann. Inst. Fourier 22 (1972).

2. L. Hörmander, An introduction to complex analysis in several variables (Van Nostrand, 1966).

3. P. Lelong, Fonctionelles analytiques et fonctions entieres ( $n$ variables) (Montreal, 1968).

4. B. Ja. Levin, Distribution of zeros of entire functions, Amer. Math. Soc. Translations of Mathematical Monographs 5 (Providence, R.I., 1964).

5. A. Martineau, Indicatrices de croissance des fonctions entières de $N$ variables, Inventiones Math. 2 (1966), 81-86.

6. A. Martineau, Indicatrices de croissance des fonctions de $N$ variables-corrections et compléments, Inventiones Math. 3 (1967), 16-19.

7. A. Martineau, Equations differentielles d'ordre infini, Bull. Soc. Math. France 95 (1967), 109-154.

8. F. Treves, Linear partial differential equations with constant coefficients (New York, 1966).

TULANE UNIVERSITY

New OrLEANS, La 70118, U.S.A. 